\title{
Neck Metastasis from A Papillary Thyroid Cancer with no Detectable Primary Tumor in Thyroid-Case Report and Current State
}

\author{
Andres Ignacio Chala ${ }^{1 *}$, Luisa Fernanda Pérez ${ }^{1}$, Humberto Ignacio Franco ${ }^{1}$, Rafael Pava ${ }^{1}$, Alex Pava ${ }^{1}$, Carlos \\ Darío Aguilar ${ }^{1}$, John Jairo Duque ${ }^{2}$
}

${ }^{1}$ Head and Neck Service, Surgical Department, University of Caldas, Colombia

${ }^{2}$ Endocrine Service, Clinical Department, Quindio's University, Colombia

*Corresponding author: Andres Ignacio Chala, Head and Neck Service, Surgical Department, Caldas University, Manizales Caldas, Colombia.

Received Date: February 21, 2020

Published Date: March 02, 2020

\begin{abstract}
Neck metastasis from a differentiated thyroid cancer with no detectable primary in the thyroid gland is rare, with few cases reported in the literature. Due to this there is no clear consensus about its etiology, treatment or prognosis. A new case in 41-year-old women with a $3 \mathrm{~cm}$ neck metastasis is presented, she had a cystic metastasis from a papillary thyroid carcinoma with no detectable primary despite a meticulous clinical and imaging inspection. Even after surgery the primary tumor couldn't be found on the thyroid gland. She had surgery and Iodine therapy. During active surveillance a central neck compartment recurrence was detected requiring surgery. A complete revision of literature shows only 9 cases reported; the theories about its origin are not clear. It seems to have as good prognosis as its counterpart with primary found in the gland.

Keywords: Unknown primary; Thyroid cancer; Neck metastasis cancer; Papillary thyroid cancer
\end{abstract}

\section{Introduction}

Thyroid cancer is the most frequent endocrine tumor of the head and neck. Most of them are papillary thyroid cancer with an incidence of neck metastasis between 20 to $60 \%$, most of them located in the central compartment. At least $30 \%$ will have a concurrent lateral neck metastasis (level II to V) or a recurrence in the lateral neck during the follow up. It is frequent to come upon an asymptomatic cystic lateral neck metastasis from a papillary thyroid cancer usually with no clinical tumor in the thyroid gland. With the increasing and improving in resolution of thyroid ultrasound, even microscopic thyroid nodules can be found, so the identification of the primary in the thyroid usually is a not a big challenge. Small nodules up to 3-5 $\mathrm{mm}$ can be responsible of a lateral neck metastasis. Fine needle aspiration biopsy (FNAB) is being used as the main method to obtain enough cytological sample in the metastatic node and in the thyroid nodule to do the diagnosis. Some other studies as CT scan or MRI are usually unnecessary and only done if the preoperative plan changes. A PET CT in these cases are not required since the primary is identified in the thyroid. Some cases when the FNAB is insufficient may require a trucut or even an open biopsy to obtain tissue. The initial treatment is a total thyroidectomy with a comprehensive neck dissection. There are few cases reported in literature presented with a neck metastasis from a thyroid cancer with no primary identified in the thyroid neither by ultrasound nor by a pathological exam of all the thyroid excised gland, so this clinical case is another contribution to this strange tumor behavior.

\section{Case Presentation}

A 41-year-old female attended at the head a neck service with a right lateral neck node level II. She noticed it for the last year, but only recently was aware due to the progressive growing. She did not refer weight loss, dysphagia, or dysphonia. Physical evaluation 
revealed neck nodes between $1-3 \mathrm{~cm}$ in level II with no fixation and with no thyroid palpable nodule. Fiberoptic Larynx evaluation was completely normal. She has no risk factors nor radiation history. Blood thyroid test was normal. (TSH: 1,5 ui/ml). Neck ultrasound showed a round cystic and solid lymph node in level II size $2.7 \mathrm{~cm}$ (Figure 1) and three additional suspicious round neck lymph nodes with microcalcifications levels II and III between $1-3 \mathrm{~cm}$. A complete and accurate ultrasound of the thyroid gland was performed finding a normal gland with no nodes (Figure 2). A FNAB was perform on the level II node identifying irregular cells with clear nuclei, nuclear clefts and fine chromatin suggestive of papillary carcinoma metastatic to the lymph node (Figure 3). A Ct scan showed similar findings to the ultrasound and the chest Rx was normal.

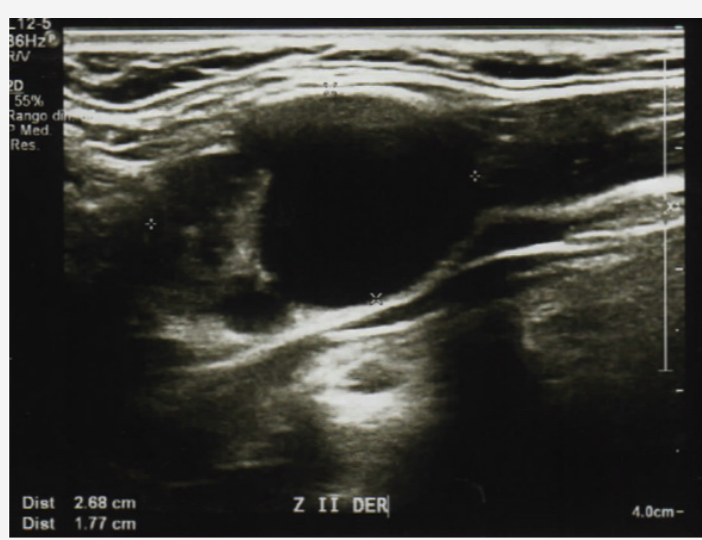

Figure 1: Ultrasound of the metastatic Lymph node showing a suspicious cystic and solid round lymph node.

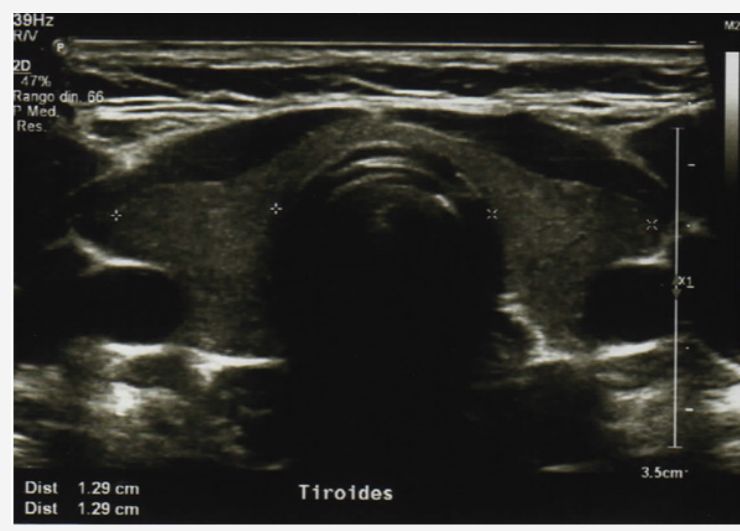

Figure 2: Normal thyroid ultrasound with no nodes.

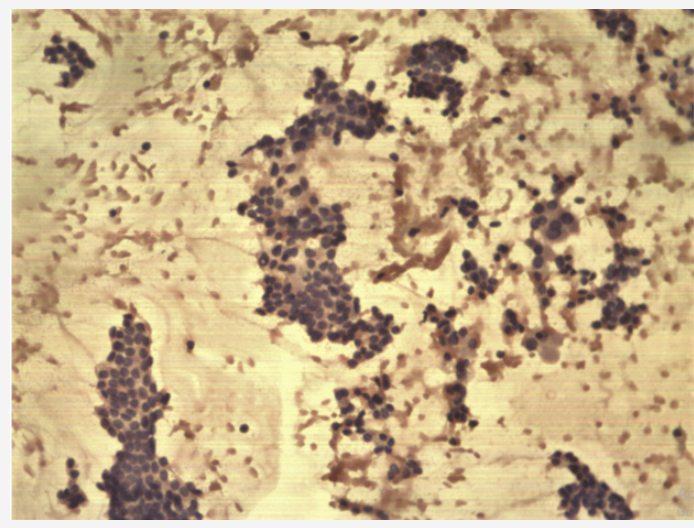

Figure 3: Fine needle aspiration biopsy of the metastatic lymph node exhibiting irregular cells with clear nuclei.

After multidisciplinary evaluation including reassessment of the FNAB, surgery was planned. Starting by a frozen biopsy that exhibited papillary structures covered by clear ovoid nuclei with clear chromatin, pseudo inclusions and microcalcifications conclusive of node metastasis from a papillary thyroid cancer. A selective neck dissection levels II to VII and a total thyroidectomy was performed. The patient was discharged the day after surgery with no complications or morbidity. The sample pathology showed 
4 nodes of level II with a classic papillary thyroid cancer size $2,5 \mathrm{~cm}$ the largest, from the total of 72 lymph nodes dissected levels II to VI. After a meticulous review of multiple sample cuts. No primary in thyroid gland was found. A new evaluation by two additional pathologist experts was done. Both agree with the diagnosis of papillary carcinoma in the lymph nodes (Figure 4) with the absence of primary in thyroid gland. The immunohistochemistry test was positive for thyroglobulin, TTF1 (Figure 5) and cytokeratin 7 (Figure 6) and negative for cytokeratin 20. The patient received $100 \mathrm{mci}$ I131 with central uptake on the scan (Figure 7) Blood test showed thyroglobulin $5.61 \mathrm{ng} / \mathrm{ml}$, antibodies antithyroglobulin 0.17 and TSH $28 \mathrm{ng} / \mathrm{ml}$. A year after treatment during active surveillance she recurred in the central neck compartment. Her thyroglobulin level was $9.7 \mathrm{ng} / \mathrm{dl}$ with negatives antibodies. Due to a confirmatory fine needle aspiration biopsy of the node, surgery with exploration in the central neck compartment was perform with no complications. Pathology showed 3 from 8 nodes between 1 to $1.5 \mathrm{~cm}$. A second $100 \mathrm{mci}$ I131 therapy showed no iodine uptake. She is on active surveillance and her blood test is now normal with thyroglobulin $0.41 \mathrm{ng} / \mathrm{ml}$, antibodies antithyroglobulin 0.19 and TSH $1.1 \mathrm{ng} / \mathrm{ml}$.

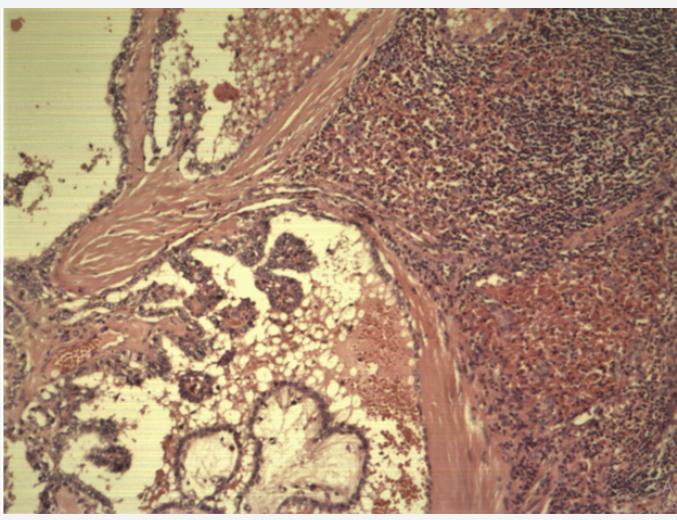

Figure 4: Pathology in the tissue of the metastatic lymph node.

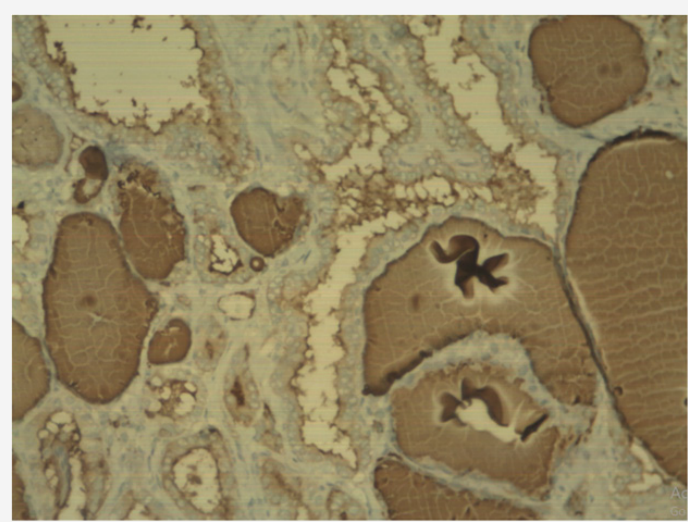

Figure 5:

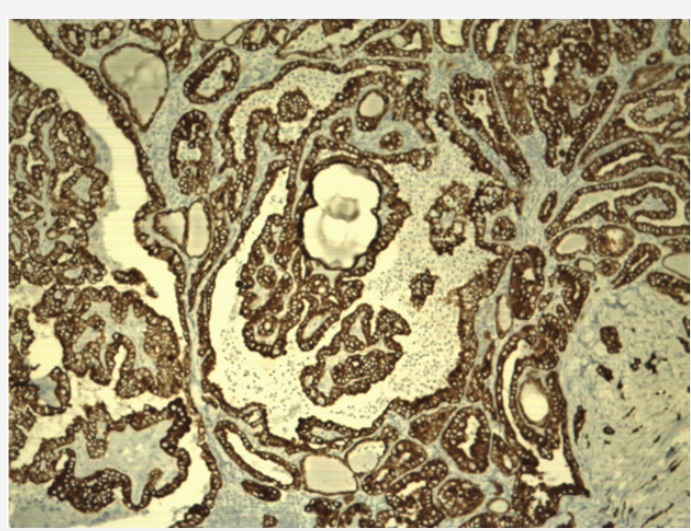

Figure 6: 


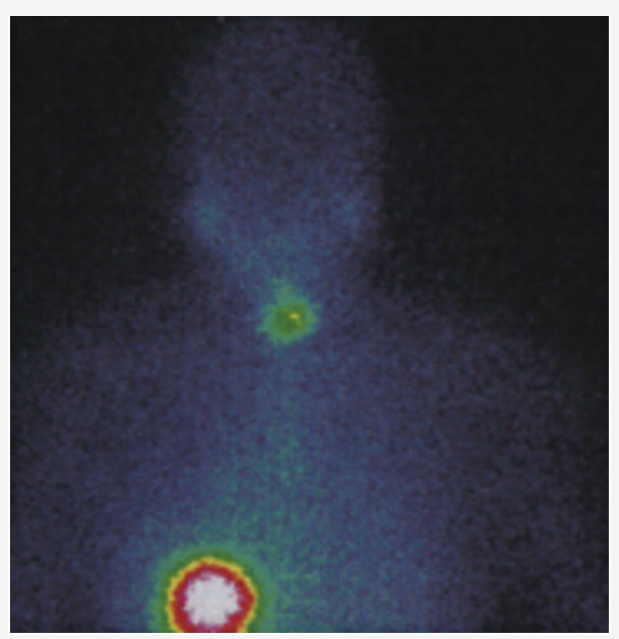

Figure 7: lodine Scan after I131 therapy showing residual central uptake with no metastatic disease.

\section{Discusion}

Papillary thyroid cancer is the most frequent malignant endocrine tumor being almost $80 \%$ of all thyroid cancer [1]. During last four decades the incidence of thyroid cancer has been increasing all over the world [2], especially due to the increased overdiagnosis of ultrasound incidentalomas. Papillary thyroid cancer usually has an excellent prognosis even the high incidence of nodal metastasis at the time of presentation. Most of them exhibit a hard-thyroid nodule and less frequently is concurrent with vocal cord paralysis due to invasion of the recurrent laryngeal nerve [3]. Nodal metastasis can be seen in almost 30 to $60 \%$ of cases, presenting as a solid or cystic metastasis or as an occult lymph metastatic node [4].

The papillary lymph node metastasis from an occult thyroid carcinoma with no structural tumor detectable in the thyroid despite the appropriate clinical or imaging evaluation is usually finally found on the histopathologic exam after the total thyroidectomy. However, in very few cases, the primary cannot be found even with a thorough and complete exam of the thyroid gland, presenting as a challenge on the diagnosis $[3,5]$. Some series report a lateral neck mass as the initial manifestation of a thyroid metastatic carcinoma, usually involving neck nodes in levels III (51\%) and IV (33\&) followed by level I (10\%) and level V (2\%) [6]. Until now, only 9 cases have been reported, all of them with a complete pathologic revision of all thyroid gland showing no primary and with a confirmed metastatic lymph node from papillary thyroid cancer [5]

There are several theories trying to explain this phenomenon. The first one is that even though the pathologist disposes of all the thyroid gland, only less than $5 \%$ of it is routinely evaluated and a microcarcinoma less than $3 \mathrm{~mm}$ could be missed even if asked specifically for searching it. Other hypothesis is a spontaneous tumoral regression, defined as a decrease of the malignancy because of immune response; this regression is suspected by the fibrosis exhibited in most of these patients. It could be supposed that the fibrosis is a sign of partial or total regression of the tumor in the thyroid cancer and that would explain this happening of metastasis without recognizable thyroid primary [5]. The last theory is an ectopic thyroid tissue in a lymph node; this rare condition defined as the presence of thyroid tissue outside pre-tracheal area has an incidence of $1-3 \%$ of all the ectopic thyroid tissue, and malignancy is only present in less than $1 \%$ of these cases. In these particular cases, its malignant transformation could be another explanation to the metastatic lymph node with no thyroid primary [7], but there is additional concern to support that theory, due to the lack of ability to differentiate between metastatic lymph node coming from a thyroid carcinoma from a malignant transformation of an ectopic thyroid tissue [7].

Most of the metastatic thyroid carcinomas can be diagnosed with the regular histopathological analysis. Few of them require immunohistochemistry tests to confirm the diagnosis. Genetic mutations are prevalent on thyroid cancer. Two-thirds of patients have BRAF V600E mutations, being the most frequent mutation found on regional metastatic tumors with unknown primary arising from papillary thyroid cancer (PTC). RAS mutations are usually found in the follicular variant from PTC and are usually related to distant metastasis [5].

Current options to evaluate metastatic lymph nodes includes ultrasound, scintigraphy, computed tomography (CT) and magnetic resonance image (MRI) [8]. Ultrasound is highly sensible to find a suspicious thyroid nodule since it can detect small nodules even a $2 \mathrm{~mm}$ size, and can also distinguish characteristics such as hypoechoic, microcalcifications, tall growth, suggesting malignancy $[7,8]$. But it has low diagnostic performance to detect suspicious nodes in the retro tracheal, intrathoracic or retro clavicular area [8], where is advisable to have CT scan or MRI images. Nevertheless, the occurrence of some proven metastatic lymph nodes from thyroid carcinomas, the primary thyroid tumor cannot be found on clinical or imaging tests. On the other hand, a metastatic lymph node (>- 
$10 \mathrm{~mm}$ ), is suggested in the presence of loss of the fatty hilum, a node being irregular, round, hypoechoic, cystic, with microcalcifications or with peripheral vascularization $[1,4,9]$.

In the clinical setting FNAB of the accessible nodes should be done as the first step to reach the diagnosis, as it is easy to perform minimally invasive and cost-effective to diagnose a differentiated metastatic thyroid carcinoma [3], especially trustable in the eyes of an expert pathologist. In cases of doubt when tissue is needed, a trucut biopsy guided by ultrasound or a complete resection of the node are alternatives procedures to diagnose the metastasis. The advantage of them is the possibility to have enough tissue to realize immunohistochemistry stains or molecular tissue tests [10]. Once the diagnosis confirms malignancy, a total thyroidectomy with central neck dissection and comprehensive neck dissection should be done. In that setting and depending on final pathology and staging, including risk staging, a I131 therapy could be considered with a total body scan to detect residual uptake or distant metastasis [11] The suppression or substitution therapy with levothyroxine must also be stablished keeping in mind the main objective according to the Clinical Stage $[6,7,10]$.

\section{Conclusion}

1) Metastatic thyroid carcinoma with no primary in thyroid after a comprehensive evaluation is unusual, with few records on literature. It is considered harboring a good prognosis with a longterm survival longer than 20 years in $90 \%$ of cases.

2) Even currently its origin is unclear, there are multiple hypothesis to explain this phenomenon being the most accepted spontaneous tumor regression, the presence of a microcarcinoma less than $3 \mathrm{~mm}$ not identified on tissue evaluation during current pathologic exam or a malignant transformation of ectopic thyroid tissue.

3) Ultrasound and CT scan are the images with higher performance to the initial diagnosis but cytologic or pathologic diagnosis is necessary so that fine needle aspiration cytology or biopsy are required to adequate the diagnosis. The treatment and prognosis are similar to the usual metastatic thyroid cancer.

\section{Acknowledgement}

The authors state that subject of this case reports has given her written informed consent to publish their case, including publication of images. The subject's identity is not going to be revealed.

\section{Conflict of Interest}

There is no conflict of interest that could be perceived as prejudicing the impartiality of the research reported There is nothing to disclosure. This research did not receive any specific grant from any funding agency in the public, commercial or notfor-profit sector. No Funding Sources were necessary. Authors Contribution All the authors contribute in part or all the process of the diagnosis, treatment or follow up of the clinical case and in the development of this paper.

\section{References}

1. Chang YC, Lo WC, Lo CY, Liao LJ (2013) Occult Papillary Thyroid Carcinoma Initially Presenting as Cervical Cystic Lymph Node Metastasis: Report of Two Cases. Journal of Medical Ultrasound Volume 21(2): 92-96.

2. Yongfu Z, Ziyu L, Chen L, Jingchao X (2018) Cervical mass as the initial manifestation of occult papillary thyroid carcinoma: Report of three cases. J Cancer Res Ther 14: S544-548.

3. Singh A, Butuc R, Lopez R (2013) Metastatic papillary thyroid carcinoma with absence of tumor focus in thyroid gland. Am J Case Rep 14: 73-75.

4. Cracchiolo JR, Wong RJ (2017) Management of the lateral neck in well differentiated thyroid cancer. Eur J Surg Oncol 44(3): 332-337.

5. Xu B, Scognamiglio T, Cohen PR, Prasad ML, Hasanovic A, et al. (2017) Metastatic thyroid carcinoma without identifiable primary tumor within the thyroid gland: a retrospective study of a rare phenomenon. Hum Pathol 1(65): 133-139.

6. Machado NO, Chopra PJ, Al Hamdani A (2009) Papillary Carcinoma of the Thyroid Presenting Primarily as Cervical Lymphadenopathy: An approach to management. Sultan Qaboos Univ Med J 9(3): 328-332.

7. Agosto Vargas Y, Gutiérrez M, Martínez JH, Mangual-Garcia M, Palermo C, et al. (2017) Papillary Thyroid Carcinoma: Ectopic Malignancy versus Metastatic Disease. Case Rep Endocrinol: 9707031.

8. Ito Y, Hirokawa M, Fukushima M, Inoue H, Yabuta T, et al. (2008) Occult Papillary Thyroid Carcinoma: Diagnostic and Clinical Implications in the Era of Routine Ultrasonography. World J Surg 32(9): 1955-1960.

9. Melany M, Chen S (2017) Thyroid Cancer. Endocrinol Metab Clin North Am 46(3): 691-711.

10. Monchik JM, De Petris G, De Crea C (2001) Occult papillary carcinoma of the thyroid presenting as a cervical cyst. Surgery 129(4): 429-432.

11. Loevner LA, Kaplan SL, Cunnane ME, Moonis G (2008) Cross-Sectional Imaging of the Thyroid Gland. Neuroimaging Clin N Am 18(3): 445-461. 